\title{
A compliance feedforward scheme for a class of LTV motion systems
}

\author{
Nikolaos Kontaras ${ }^{1}$, Marcel Heertjes ${ }^{2}$, Hans Zwart ${ }^{3}$, and Maarten Steinbuch ${ }^{4}$
}

\begin{abstract}
The implementation of lightweight highperformance motion systems in lithography applications imposes among others lower requirements on actuators, amplifiers, and cooling. However, the decreased stiffness of lightweight designs brings the effect of structural flexibilities to the fore especially when the so-called point of interest is not at a fixed location. This is for example the case when exposing a silicon wafer. To deal with structural flexibilities, a feedforward controller is proposed that combines two concepts: (a) continuous compliance compensation control and (b) snap feedforward control. Expanded to a subclass of LTV motion systems, the resulting controller compensates for the position-dependent and time-varying compliance of a flexible structure. The compliance function used will be derived using partial differential equations (PDE). The method is validated by simulation results.
\end{abstract}

\section{INTRODUCTION}

In the semiconductor industry, the focus on ever improving throughput, overlay, and imaging of exposed silicon wafers traditionally lead to more aggressive motion profiles, i.e. higher accelerations, and structural designs with higher stiffness and mass. The required forces to be applied during operation are therefore increasingly higher, which increases the demands on actuators, amplifiers, and cooling. The resulting force density and heat generation necessitated to accelerate the mass therefore becomes increasingly infeasible, which prompts for more flexible lightweight designs [14].

As a consequence, when the performance location changes with time which typically occurs during wafer exposure, the dynamics of the system are expressed differently due to the different contributions from structural modes at that specific location. This leads to the requirement of taking the timevarying aspect of the plant into account when calculating feedforward compensation forces, which are key in achieving position accuracy.

The traditional approach toward the positioning problem would be an acceleration (or mass) feedforward controller.

\footnotetext{
${ }^{1}$ Nikolaos Kontaras is with the Department of Mechanical Engineering, Control Systems Technology group , Eindhoven University of Technology, 5612 AZ Eindhoven, The Netherlands n. kontarasetue.nl

${ }^{2}$ Marcel Heertjes is with the Department of Mechanical Engineering, Control Systems Technology group, Eindhoven University of Technology, 5612 AZ Eindhoven, The Netherlands m. f.heert jes@tue.nl

${ }^{3}$ Hans Zwart is with the Department of Mechanical Engineering, Dynamics and Control group, Eindhoven University of Technology, 5612 AZ Eindhoven, The Netherlands h.j.zwart@tue.nl, and with the Faculty of Electrical Engineering, Mathematics and Computer Science, Department of Applied Mathematics, University of Twente, 7500 AE Enschede, The Netherlands h.j.zwarteutwente.nl

${ }^{4}$ Maarten Steinbuch is with the Department of Mechanical Engineering, Control Systems Technology group, Eindhoven University of Technology, 5612 AZ Eindhoven, The Netherlands m. steinbuch@tue.nl
}

But this is not sufficient to compensate for the flexible dynamics. Alternatively, snap feedforward control [9] can only account for structural flexibilities to a certain extend, and cannot cope easily with time or parameter-varying dynamics. In [4] and [5] a collection of feedback and feedforward control methods are summarized, applicable to non-minimum phase and flexible motion systems. Among them, the work in [7] proposes a model inverse-based feedforward control signal for nonlinear plants, which requires the feedforward signal be known a priori and also pre-actuation, i.e. noncausal control effort. More recently, [8] addresses the problem of regulating the plant output when the disturbance is not known a priori, and [12] proposes a model-based non-causal feedforward scheme for double integrator-based Linear Time-Varying (LTV) systems, a class of systems also considered in the present work. In [6], work has been done on the discrete-time control of a stage, considering the plant as a Linear Time-Invariant (LTI) system. In [11] a feedforward method for flexible systems with time-varying performance locations is presented. The method utilizes a lifted feedforward (discrete-time) representation, however, it does not take the plant variation in-between the timeintervals into account. Spatial feedforward control [10] has been developed in order to prevent excitation of the structural modes of the positioning system. However this method uses over-actuation. Hence, the number of structural modes to be suppressed should equal the number of additional actuators.

Different from the current approaches in the literature, this work introduces a compliance compensating feedforward control scheme for motion systems with time-varying performance locations. That is, the same system class as addressed in [13]. The feedforward controller proposed here will be combined with the control objectives of classical snap feedforward, but for a time-varying performance location. Therefore this feedforward controller can account for position-dependent dynamics, which does not merely attempt at masking possible internal chuck deformations (ICD) caused during acceleration and deceleration, but instead generates appropriate control effort (force) to counteract such deformations. This renders the controller capable of tackling more arbitrary tracking set-points and control objectives than in [13]. Furthermore, as low as second-order set-points are compatible with this control scheme, which is the minimum required to be followed by a mass. Moreover, third order set-points produce a continuous control effort while fourthorder or higher set-points produce a smooth control effort which doesn't excite higher order dynamics as much as snap feedforward control. This feedforward controller does not require pre-actuation, i.e. is causal, which allows for set- 
points not given a priori. The spatially continuous dynamics of the plant used as an example in this work (a flexible Euler-Bernoulli beam) are derived from the partial differential equation (PDE) representation, and so is the positiondependent compliance function of the beam. The method is validated by continuous-time simulation.

The remainder of this paper is organized as follows. Section II introduces the problem statement. Section III proposes the novel feedforward control scheme, along with its mathematical derivation, notions of stability and performance. Section IV discusses the simulation environment and the results which validate the method. Finally, in Section V, some concluding remarks are given.

\section{Problem Statement}

During the production of chips, a silicon wafer is positioned atop the wafer stage of a lithographic system. A source emanating (extreme) ultraviolet (EUV) light passes through the reticle, which is part of the reticle stage, and which contains a blueprint of the integrated circuits (ICs) to be processed. Beyond the reticle, light passes an optical column with projection lenses before it exposes the photo-sensitive layers of the wafer's surface. An illustration of the wafer stage during exposure is shown in Fig. 1. Assuming that it is a lightweight structure, i.e. its dynamic behavior is substantially dependent on position, it follows that during exposure the time-varying performance location is subjected to position-dependent dynamics.

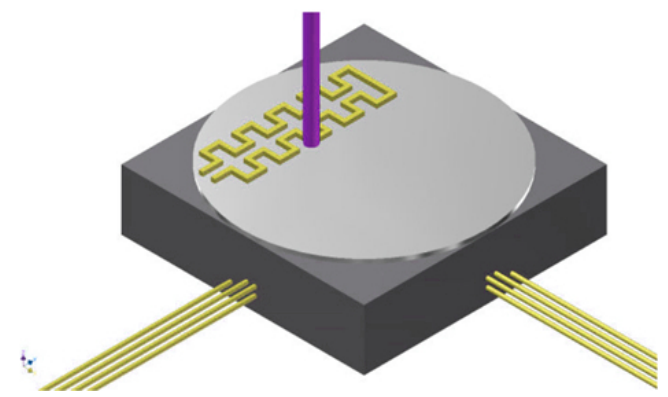

Fig. 1: Schematic representation of a wafer stage of a lithographic system, where the sensors lie at the edges of the stage, and where during exposure of the silicon wafer to the laser beam, the performance location changes over time.

Consider a straightforward snap feedforward control scheme [9], illustrated in Fig. 2, and an LTI double-integrator based motion system $P$, that is,

$$
P(s)=\underbrace{\frac{1}{m s^{2}}}_{P_{\mathrm{rb}}}+\underbrace{\sum_{n=1}^{l} \frac{b_{0, n} c_{0, n}}{m_{n} s^{2}+d_{n} s+k_{n}}}_{P_{\mathrm{nrb}}},
$$

where $m$ is the total mass, $b_{0, n}$ and $c_{0, n}$ the input and output coefficients, $m_{n}, d_{n}$ and $k_{n}$ the modal mass, damping and stiffness respectively, $P_{\mathrm{rb}}$ the rigid body (RB) mode, $l \in \mathbb{N}^{+}$the number of non-rigid body modes (NRB), and $s$ the Laplace variable. Aiming to satisfy the control objective of perfectly compensating for the low-frequency properties of the plant, i.e. mass and compliance, the snap feedforward controller follows from the principle of plant inversion, or

$$
F F_{\text {snap }}(s)=\frac{U_{f f}(s)}{Y_{d}(s)}=K_{f a} s^{2}+K_{f s} s^{4}=m s^{2}-m^{2} C s^{4},
$$

with $Y_{d}(s), U_{f f}(s)$ the Laplace transforms of the desired output and the control effort respectively, and $C$ the compliance, which equals the DC contributions of all the NRB modes of $P_{n r b}$, that is,

$$
C=\sum_{n=1}^{l} \frac{b_{0, n} c_{0, n}}{k_{n}} .
$$

A straightforward attempt to extend this control scheme for

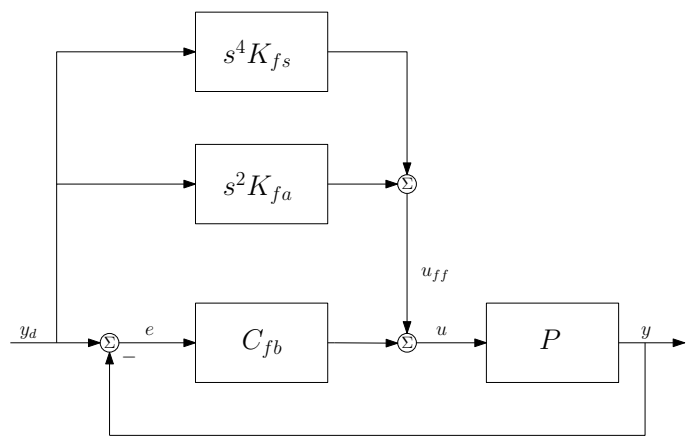

Fig. 2: Snap feedforward control scheme.

plants with a now time-varying compliance function, defined as $C(t)$, is to straightforwardly use the feedforward controller

$$
F F_{\text {snap }}(p, t)=\frac{u_{f f}(t)}{y_{d}(t)}=m p^{2}-m^{2} C(t) p^{4},
$$

where $p=\mathrm{d} / \mathrm{d} t$ is the time differential operator ${ }^{1}$. It will be shown however that in general, feedforward control schemes developed for LTI systems such as (2) cannot be directly applied to position-dependent and time-varying systems.

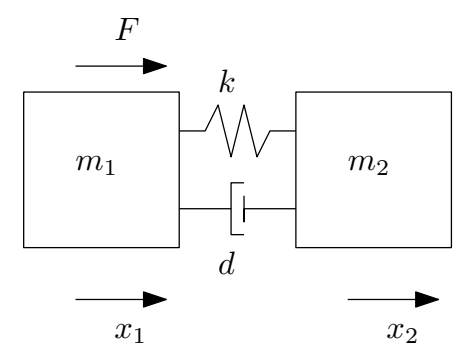

Fig. 3: Mass-spring-damper (MSD) system.

To see this, consider the example of a mass-spring-damper LTI Single-Input Multiple-Output (SIMO) system, shown in Fig. 3. Given no damping, i.e. $d=0$, the non-collocated (from force $F$ to displacement $x_{2}$ ) response of the system is given by

$$
P_{\mathrm{msd}}(s)=\frac{k}{s^{2}\left(m_{1} m_{2} s^{2}+k\left(m_{1}+m_{2}\right)\right)} .
$$

\footnotetext{
${ }^{1}$ Use of $p$ instead of $s$ is required due to the time-varying nature of the controller, which does not admit a straightforward Laplace transform.
} 
The total mass of the system equals $m_{\mathrm{msd}}=m_{1}+m_{2}$ and from (5) it can be found that the compliance is

$$
C_{\mathrm{msd}}=-\frac{m_{1} m_{2}}{m_{\mathrm{msd}}^{2} k} \text {. }
$$

As follows from (2) and (5), the snap feedforward controller

$$
F F_{\text {snap-msd }}(s)=m_{\mathrm{msd}} s^{2}-m_{\mathrm{msd}}^{2} C_{\mathrm{msd}} s^{4}
$$

perfectly accounts for the plant dynamics in the sense that $P_{\text {msd }}(s) F F_{\text {snap-msd }}(s)=1$.

Let us now consider by means of example a smooth timevarying stiffness, i.e. $k(t) \in \mathcal{C}^{2}$.This introduces a timevarying compliance in (5), while the mass of the system remains the same. The equations of motion are given by

$$
\begin{aligned}
F(t)-k(t)\left(x_{1}(t)-x_{2}(t)\right) & =m_{1} \ddot{x}_{1}(t) \\
k(t)\left(x_{1}(t)-x_{2}(t)\right) & =m_{2} \ddot{x}_{2}(t) .
\end{aligned}
$$

Assuming a desired trajectory for the second mass, $y_{d}(t)$, eliminating $x_{1}(t)$ from (8), the force $F_{\mathrm{msd}}(t)$ required to account for the plant dynamics is given as follows,

$$
\begin{aligned}
F_{\mathrm{msd}}(t)= & m_{\mathrm{msd}} \ddot{y}_{d}(t)+\frac{2 \dot{k}^{2}(t)-k(t) \ddot{k}(t)}{k^{3}(t)} m_{1} m_{2} \ddot{y}_{d}(t) \\
& -\frac{\dot{k}(t)}{k^{2}(t)} 2 m_{1} m_{2} y_{d}(t)^{(3)}+\frac{m_{1} m_{2}}{k(t)} y_{d}(t)^{(4)} .
\end{aligned}
$$

The first and last term of (9) correspond to the mass and snap terms in (7), however as it can be seen there are two additional terms required in order to account for the timevarying plant dynamics. In (9), the first two time derivatives of the time-varying stiffness appear, along with the third time-derivative of $y_{d}(t)$. Therefore, the structure as suggested by (4) is inherently too limited to cope with the time-varying stiffness.

To account for time-varying compliance, we first adopt the more general (possibly infinite-dimensional) motion system $P$, described by the LTV state-space model,

$$
\begin{aligned}
& \dot{x}(t)=A^{\prime} x(t)+B^{\prime} u(t), \\
& y(t)=C^{\prime}(t) x(t)+D^{\prime}(t) u(t),
\end{aligned}
$$

where $A^{\prime} \in \mathbb{R}^{n \times n}, B^{\prime} \in \mathbb{R}^{n \times 1}, C^{\prime}(t) \in \mathbb{R}^{1 \times n}, D^{\prime}(t) \in$ $\mathbb{R}^{1 \times 1}$. The output matrices can be time-dependent, due to a time-varying point-of-interest.

In terms of (1), the symbolic transfer function from $u$ to $y$ of (10) can be written as

$$
P(p, t)=\frac{y(p, t)}{u(p, t)}=\frac{1}{m p^{2}}+\sum_{n=1}^{l} \frac{b_{0, n} c_{0, n}(t)}{m_{n} p^{2}+d_{n} p+k_{n}} .
$$

\section{COMPLIANCE FEEDFORWARD CONTROL FOR A CLASS OF LTV MOTION SYSTEMS}

The proposed control scheme is illustrated in Fig. 4 and consists of the following components,

1) Desired output signal; the signal $y_{d}$ can be a secondorder or higher setpoint, which typically has a continuous first derivative (velocity), that is $y_{d} \in \mathcal{C}^{1}(0, \infty)$;

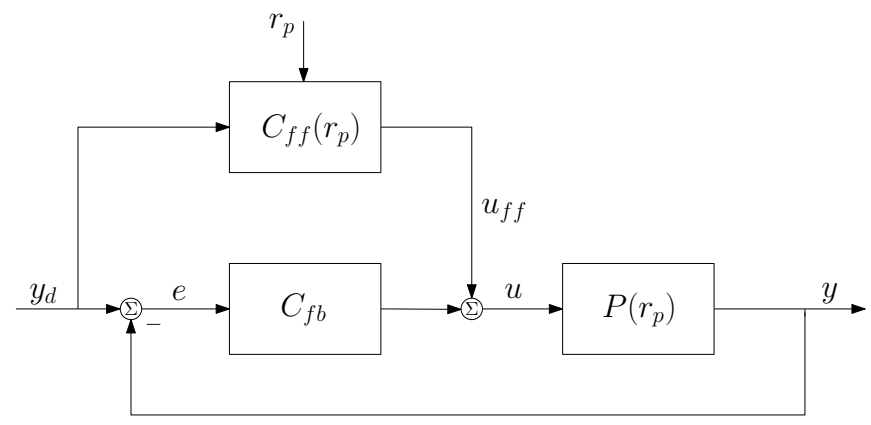

Fig. 4: Block diagram of the proposed LTV feedforward control scheme.

note that for the lithographic industry the scanning interval of constant velocity (in-between the dashed lines) is the interval in which the tracking error is required to be sufficiently small;

2) Performance location function; in the case of positiondependent dynamics, a real-valued function $r_{p}=r_{p}(t)$ is required, which indicates the point of interest (POI) as a function of time $t \in \mathbb{R}$; for a distributed parameter system, $r_{p}$ is continuously differentiable at least once, i.e. $r_{p} \in \mathcal{C}^{1}$; note that in some applications $r_{p}=y_{d}$, e.g. during wafer stage operation, in the $x$ and $y$ directions, which are parallel to the stage;

3) Plant; the plant $P\left(r_{p}\right)$ is a Single-Input Single-Output (SISO) flexible motion system, defined by (10), or "equivalently" by (11); its performance location can be static or time-varying in nature indicated by $r_{p}$;

4) Feedback controller; the LTI feedback controller $C_{f b}$ acts on the error $e$ between the setpoint and the plant output, i.e. $e=y_{d}-y$;

5) Feedforward controller; the feedforward controller $C_{f f}$ accounts for the mass and time-varying compliance of $P$; it connects the setpoint $y_{d}$ to the output signal $u_{f f}$.

\section{A. Controller derivation}

Consider a system as in (1). A low-frequency approximation can be derived by preserving the RB mode, i.e. $P_{r b}(s)$, plus the total compliance, the latter is given in in (3). Assuming the input and output signals admit Laplace transformations $U(s), Y(s)$, the low-frequency approximation is given by

$$
\begin{aligned}
P_{0,1}(s)=\frac{Y(s)}{U(s)} & =P_{r b}(s)+C \\
& =\frac{1}{m s^{2}}+\sum_{n=1}^{l} \frac{b_{0, n} c_{0, n}}{k_{n}} .
\end{aligned}
$$

Given a time-varying compliance function $C=C(t)$, the input-output relation in time-domain can be represented by

$$
\begin{array}{r}
P_{0,2}(p)=\frac{y(t)}{u(t)}=\frac{1}{m p^{2}}+C(t) \Leftrightarrow \\
y=\frac{1}{m} \iint u(\tau) \mathrm{d} \tau+C(t) u(t) \Leftrightarrow \\
m \ddot{y}(t)=u(t)+m(C(t) \ddot{u} u(t)) .
\end{array}
$$


The plant in (13) provides an accurate plant approximation in the low frequency interval only. Plant inversion of (13) would (if possible) produce a model-based feedforward controller which can account for low-frequency output disturbances, i.e. tracking setpoints commonly used in such systems. Assuming $y_{d}$ a desired output trajectory and $u_{f f}$ the desired control input, yields the feedforward control differential equation

$$
\ddot{u}_{f f}(t)=-\frac{2 \dot{C}(t)}{C(t)} \dot{u}_{f f}(t)-\frac{1+m \ddot{C}(t)}{m C(t)} u_{f f}(t)+\frac{1}{C(t)} \ddot{y}_{d} .
$$

As it can be seen, a critical issue in solving (14) is the division by the compliance function $C(t)$. This results in the possibility of division by zero, namely if the compliance function becomes zero, as found in [13]. Furthermore, even if $C(t)$ is non-zero and slowly varying, it can be shown that the solution to (14) is only marginally stable if $C(t)<0$, causing undesirable undamped oscillations.

To resolve the possible non-feasibility of the inversion, a plant modification is introduced which allows for inversion and simultaneously maintains a fairly accurate low frequency plant description. This is achieved by pre-filtering the timevarying compliance function $C(t)$ with a second-order lowpass filter, as shown in Fig. 5. Note that a second (or arbitrarily higher) order low-pass filter is required to prevent division by zero when solving for the signal $v(t)$. The plant

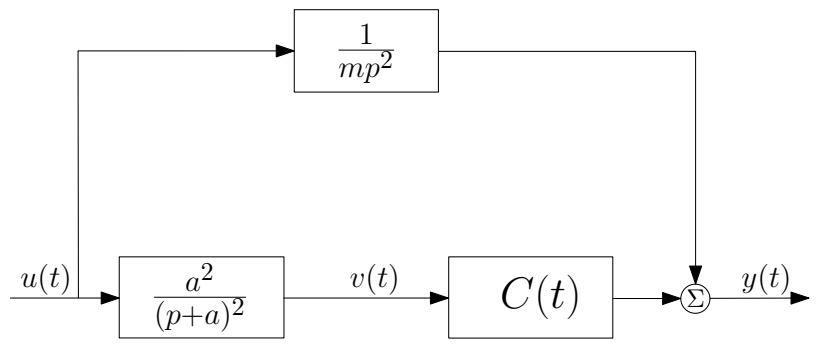

Fig. 5: Plant approximation $P_{2}(p)$ underpinning the proposed feedforward controller, consisting of a RB mode, and secondorder low-pass filter cascaded with a time-varying gain $C(t)$ which equals the time-varying compliance of the system.

to be inverted as illustrated in Fig. 5 is governed by the equations

$$
\begin{array}{r}
y(t)=C(t) v(t)+\frac{1}{m} \iint^{t} u(\tau) \mathrm{d} \tau \Leftrightarrow \\
m \ddot{y}(t)=u(t)+m(C(t) \ddot{v}(t)),
\end{array}
$$

and

$$
a^{2} u(t)=\ddot{v}(t)+2 a \dot{v}(t)+a^{2} v(t),
$$

where $a>0$ denotes the cut-off frequency of the low-pass filter. Given a desired trajectory $y_{d}(t) \in \mathcal{C}^{1}$, solving (16) with respect to $v(t)$ and after substitution in (15), gives the differential equation,

$$
\begin{aligned}
\ddot{v}(t)=\underbrace{-\frac{2 a(a m \dot{C}(t)+1)}{a^{2} m C(t)+1}}_{\xi_{1}(t)} \dot{v}(t) & \underbrace{-\frac{a^{2}(m \ddot{C}(t)+1)}{a^{2} m C(t)+1}}_{\xi_{2}(t)} v(t) \\
& \underbrace{+\frac{a^{2} m}{a^{2} m C(t)+1}}_{\xi_{3}(t)} \ddot{y}_{d}(t) .
\end{aligned}
$$

Since there is no explicit solution to (17), the signals $v(t)$, $\dot{v}(t)$, and $\ddot{v}(t)$ for a given $y_{d}$ are obtained through numerical integration. Utilizing (16) then gives the control input by

$$
u_{f f}(t)=\underbrace{\frac{1}{a^{2}}}_{\mu_{1}} \ddot{v}(t)+\underbrace{\frac{2}{a}}_{\mu_{2}} \dot{v}(t)+v(t) .
$$

In state-space form, the feedforward controller can be written and implemented as

$$
\begin{gathered}
\dot{x}(t)=\underbrace{\left[\begin{array}{cc}
0 & 1 \\
\xi_{2}(t) & \xi_{1}(t)
\end{array}\right]}_{A_{F F}(t)} x(t)+\underbrace{\left[\begin{array}{c}
0 \\
\xi_{3}(t)
\end{array}\right]}_{B_{F F}(t)} \ddot{y}_{d}(t), \\
u_{f f}(t)=\underbrace{\left[\begin{array}{cc}
\mu_{1} \xi_{2}(t)+1 & \mu_{1} \xi_{1}(t)+\mu_{2}
\end{array}\right]}_{C_{F F}(t)} x(t) \\
+\underbrace{\left[\mu_{1} \xi_{3}(t)\right]}_{D_{F F}(t)} \ddot{y}_{d}(t),
\end{gathered}
$$

where $x(t)=\left[\begin{array}{ll}x_{1}(t) & x_{2}(t)\end{array}\right]^{T}=[v(t) \dot{v}(t)]^{T}$. Naturally, in the context of this control scheme, time-dependency of the compliance function and its derivatives is introduced by the POI function $r_{p}(t)$, thus yielding $C\left(r_{p}(t)\right), \dot{C}\left(r_{p}(t)\right)$, and $\ddot{C}\left(r_{p}(t)\right)$. A block diagram of this feedforward control scheme is depicted in Fig. 6. Note that the second timederivative of $y_{d}$ is often known a priori.

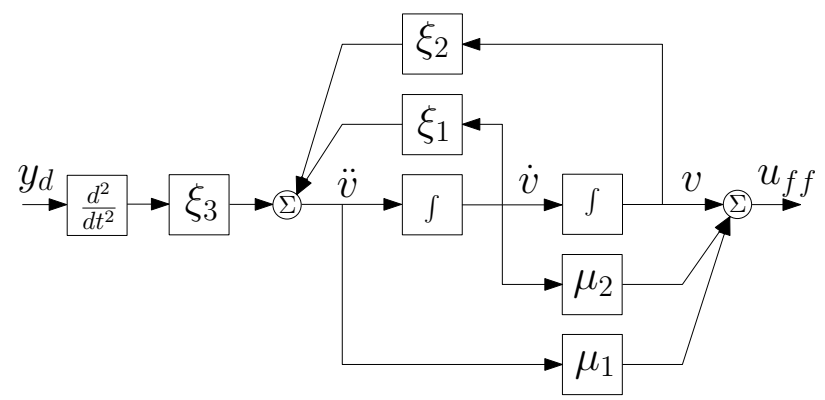

Fig. 6: Compliance compensating feedforward scheme.

\section{B. Feasibility analysis}

In terms of feasibility, as previously mentioned, division by zero mid-experiment should be excluded, otherwise (17) cannot have a solution. Moreover, for LTI cases, (17) needs to be asymptotically stable in order for the feedforward controller to be bounded-input, bounded-output (BIBO) stable. 
Following this reasoning, $a$ can be chosen as follows,

$$
\begin{cases}a \in \mathbb{R}^{+} & \text {if } C(t)>0 \\ a<\frac{1}{\sqrt{-M C(t)}} & \text { if } C(t)<0\end{cases}
$$

which also translates to keeping the eigenvalues of $A_{F F}(t)$ inside the left-half (complex) plane (LHP).

\section{NUMERICAL RESULTS}

The simulation results given in this section illustrate the compliance compensating feedforward control scheme when applied to a beam system with a time-varying performance location. For the plant, an Euler-Bernoulli beam is considered [1][2], which is illustrated and specified in Fig. 7. These specifications induce a frequency response that has similarities to a typical wafer stage system. That is with respect to both the RB and the first NRB mode's frequency and magnitude.

In frequency domain, the PDE describing this beam is given by,

$$
\frac{d^{4}}{d r_{p}^{4}} Y\left(s, r_{p}\right)+\frac{2325}{3 s+510^{8}} s^{2} Y\left(s, r_{p}\right)=0 .
$$

The solution to (21) yields the position-dependent transfer function of the beam, $G_{d}\left(s, r_{p}\right)$ [13]. This infinitedimensional transfer function can be expanded via modal approximation into its RB and infinite NRB modes, bringing it to the form that closely matches (11) but still being of infinite order. For simulation purposes, a finite-order model can be obtained through truncation. In this simulation model the RB and the first NRB mode of the beam will be included. It should however, be mentioned that the modeling strategy remains valid if more than one NRB modes are considered.

Given a pole $\lambda_{k}$, we define $a=\operatorname{Re}\left(\lambda_{k}\right), b=\operatorname{Im}\left(\lambda_{k}\right)$, $c\left(r_{p}\right)=\operatorname{Re}\left(\operatorname{Res}\left(\lambda_{k}, r_{p}\right)\right), d\left(r_{p}\right)=\operatorname{Im}\left(\operatorname{Res}\left(\lambda_{k}, r_{p}\right)\right)$, where $\operatorname{Res}\left(\lambda_{k}, r_{p}\right)$ is the Cauchy residue of the pole $\lambda_{k}$ (see [3]), which is dependent on the point of interest function $r_{p}$. The first NRB mode of the beam is given by

$$
\begin{aligned}
G_{1}\left(s, r_{p}\right) & =\frac{\operatorname{Res}\left(\lambda_{1}, r_{p}\right)}{s-\lambda_{1}}+\frac{\operatorname{Res}\left(\lambda_{1}^{*}, r_{p}\right)}{s-\lambda_{1}^{*}} \\
& =\frac{2\left(c\left(r_{p}\right) s-a c\left(r_{p}\right)-b d\left(r_{p}\right)\right)}{s^{2}-2 a s+a^{2}+b^{2}} .
\end{aligned}
$$

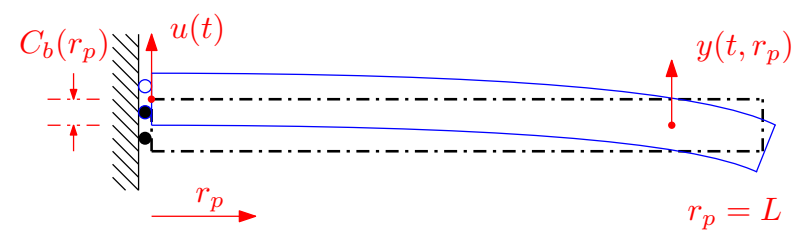

Fig. 7: Vertically-moving cantilever Euler-Bernoulli beam, where $u(t)$ the actuation force, $y\left(t, r_{p}\right)$ the displacement at the point-of-interest $r_{p}$, and $C_{b}\left(r_{p}\right)$ the position-dependent compliance; length $L=0.6[\mathrm{~m}]$, cross-sectional area $A=$ $h^{2}=10^{-4}\left[\mathrm{~m}^{2}\right]$, mass density $\rho=7.75 \cdot 10^{3}\left[\mathrm{~kg} / \mathrm{m}^{3}\right]$, Young's modulus $E=2 \cdot 10^{3}\left[\mathrm{~kg} /\left(\mathrm{m} \cdot \mathrm{sec}^{2}\right)\right]$, second moment of area $I=h^{4} / 12=10^{-4} / 12\left[\mathrm{~m}^{4}\right]$, Kelvin-Voigt damping $c_{d}=10^{-3}$.
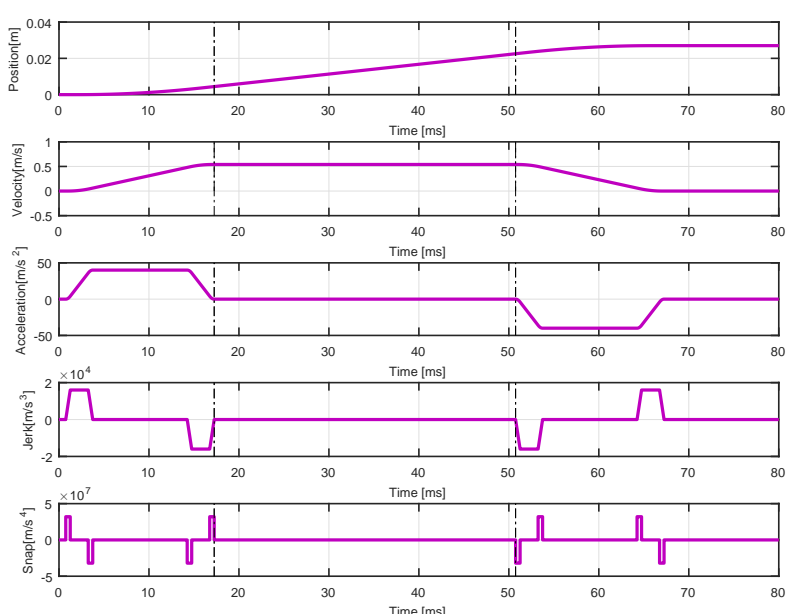

Fig. 9: Fourth-order reference setpoint $y_{d}$. The black dashed lines enclose the critical scanning interval (constant velocity).

Naturally the frequency response along the beam changes only through the residue, which affects only the zeros. The poles of the structure remain unchanged, regardless of the point of interest $r_{p}=r_{p}(t)$. From (22) it can be seen that the compliance of the single flexible mode is given by,

$$
C_{\lambda_{1}}\left(r_{p}\right)=\lim _{s \rightarrow 0} G_{1}\left(s, r_{p}\right)=\frac{-2\left(a c\left(r_{p}\right)+b d\left(r_{p}\right)\right)}{a^{2}+b^{2}} .
$$

Generally, the compliance function of the beam does not equal the compliance of its first NRB mode. Therefore an adjustment in the compliance function is required to correctly match the compliance to the infinite-dimensional beam system, similar to the approximation in (12), however now one NRB mode is preserved. The simulation model is given as follows,

$$
G_{\mathrm{s}}\left(s, r_{p}\right)=G_{\mathrm{rb}}(s)+G_{1}\left(s, r_{p}\right)-C_{\lambda_{1}}\left(r_{p}\right)+C_{b}\left(r_{p}\right),
$$

where the RB mode is given by

$$
G_{\mathrm{rb}}(s)=\frac{1}{\rho A L s^{2}},
$$

and

$$
C_{\mathrm{b}}\left(r_{p}\right)=\frac{6 L^{4}-30 L^{2} r_{p}^{2}+20 L r_{p}^{3}-5 r_{p}^{4}}{120 E I L},
$$

is the compliance function of the beam, see [13].

Simulations were performed in continuous time. The fourth-order trajectory $y_{d}$ in Fig. 9 is used as the tracking setpoint, in order to facilitate a comparison with snap feedforward. Two example POI functions $r_{p}$ are chosen, one staying in the area of the beam where $C\left(r_{p}\right)>0$, and the other one also venturing toward the area where $C\left(r_{p}\right)<0$ :

$$
r_{p_{1}}(t)=0.1(1-\cos (12.5 \pi t)),
$$

and

$$
r_{p_{2}}(t)=0.3(1-\cos (12.5 \pi t)) \text {. }
$$




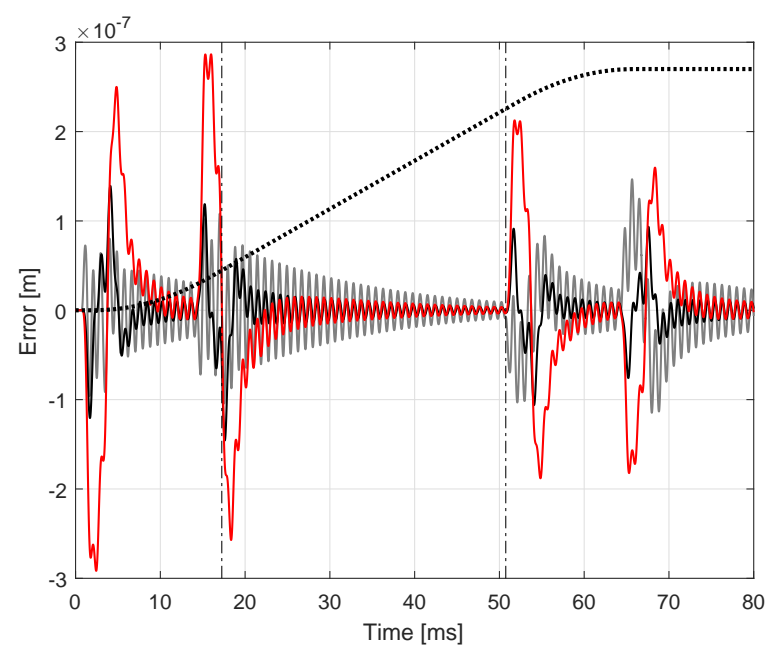

(a) POI function $r_{p_{1}}(t)$

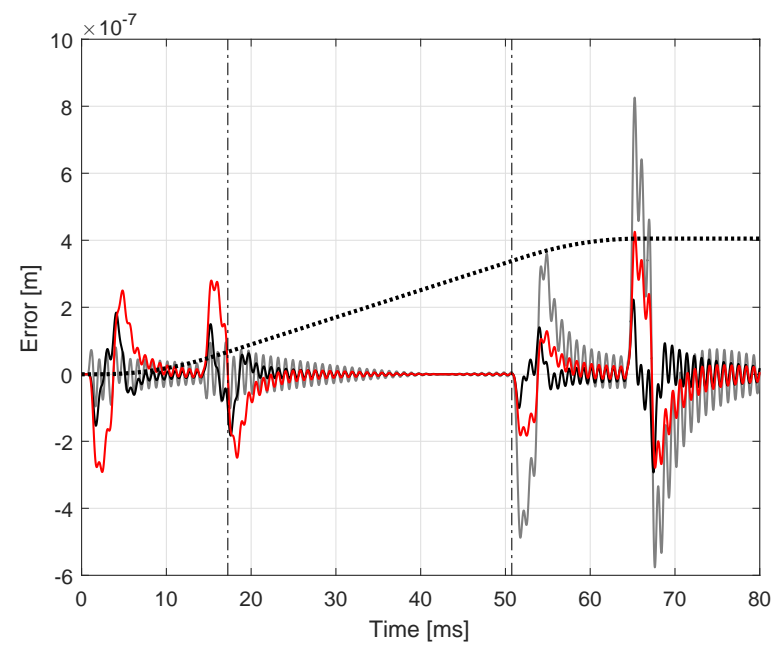

(b) POI function $r_{p_{2}}(t)$

Fig. 8: Simulation with position-dependent (black), (fixed) snap (gray) and (fixed) acceleration feedforward (red) for the beam with a time-varying performance location, in closed loop; the scanning interval is enclosed by the vertical dashed lines, while the (scaled) setpoint is drawn in dotted curves.

The feedback controller, which robustly stabilizes the beam in the case that one or two NRB modes are included in the model, irrespective of the position $r_{p}$ considered, is given by

$$
C_{\mathrm{fb}}(s)=C_{\mathrm{PID}}(s) C_{1 \mathrm{st}}(s) N_{1}(s) N_{2}(s) N_{\mathrm{s}}(s),
$$

where

$$
\begin{gathered}
C_{\mathrm{PID}}(s)=\frac{1.6410^{8} s^{2}+6.4810^{10} s+9.9310^{11}}{s}, \\
C_{1 \mathrm{st}}(s)=\frac{1}{s+2.08810^{5}}, \\
N_{1}(s)=\frac{s^{2}+0.3184 s+5.19110^{7}}{s^{2}+1.29710^{4} s+5.19110^{7}}, \\
N_{2}(s)=\frac{s^{2}+8.823 s+1.51610^{9}}{s^{2}+7.00810^{4} s+1.51610^{9}},
\end{gathered}
$$

and

$$
N_{\mathrm{s}}(s)=\frac{6.2510^{-6} s^{2}+7.226 s+2.08810^{8}}{s^{2}+2890 s+2.08810^{8}} .
$$

For the case $r_{p_{1}}$ in (27), a cut-off frequency $a_{1}=$ $7400[\mathrm{rad} / \mathrm{sec}]$ was chosen, and for $r_{p_{2}}$ in (28) the cut-off frequency is $a_{2}=4700[\mathrm{rad} / \mathrm{sec}]$.

The simulation results are illustrated in Fig. 8, which show the tracking error in closed loop using the proposed feedforward controller (black), a fixed acceleration feedforward (red), and a position-independent (or fixed) snap feedforward controller (gray), which was tuned to account for the compliance at the base of the beam, i.e. $C_{b}(0)$ in (26). It can be seen that the proposed feedforward controller results in a smaller error when compared both to snap feedforward and acceleration feedforward control. Superiority over acceleration feedforward control can be understood in terms of the more complex (position-dependent) model used to calculate the proposed feedforward controller, which can account for compliant part of the beam system and for which acceleration feedforward is unable to compensate. Snap feedforward control, while correctly tuned for the base of the beam, starts to deteriorate the error, observed for both POI functions. Moreover, the discontinuous, step-like control inputs produced by the snap feedforward controller lead to higher oscillations of the system, for which the smooth signal produced by the proposed LTV controller does not suffer from.

\section{CONCLUSIONS AND REMARKS}

Inspired by the snap feedforward [9] and the continuous compliance compensation scheme [13], on which an accurate compliance function was found, this paper further exploits this information. More precisely, a model-based compliance compensating feedforward controller is obtained for flexible structures with a time-varying performance location. A lowfrequency approximation of the plant was used to produce the controller, modified sufficiently in order to be invertible, while its inverse is simultaneously stable. Simulations using an Euler-Bernoulli beam's PDE representation, a fourth-order setpoint trajectory and two arbitrary point-of-interest time functions indicate improved performance in terms of the closed loop tracking error, regarding the compliant part of the LTV system. In terms of wafer stage control, this may help in reducing internal chuck deformations otherwise resulting in the controlled stage dynamics.

\section{REFERENCES}

[1] Moheimani, S.O.R., Halim, D., and Fleming, A.J. (2003). "Spatial Control of Vibration", World Scientific.

[2] Herrmann, L. (2008). "Vibration of the Euler-Bernoulli Beam with Allowance for Dampings", Lecture Notes in Engineering and Computer Science, vol. 2078, pp. 901-904.

[3] Curtain, R., and Morris, K. (2009). "Transfer Functions of Distributed Parameter Systems: A Tutorial”, Automatica, vol. 45, issue 5, ISSN 0005-1098, pp. 1101-1116. 
[4] Pao, L. Y., Butterworth, J. A., and Abramovitch, D. Y. (2007). "Combined feedforward/feedback control of atomic force microscopes", American Control Conference, 3509-3515.

[5] Butterworth, J. A., Pao, L. Y., and Abramovitch, D. Y. (2012). "Analysis and comparison of three discrete-time feedforward model-inverse control techniques for nonminimum-phase systems", Mechatronics, 22(5), 577-587.

[6] Yazaki, Y., Fujimoto, H., Sakata, K., Hara, A., and Saiki, K. (2015). "Application of mode switching control using initial state variables in constraint final-state control to high-precision dual stage", Paper presented at the Proceedings of the American Control Conference, 2015-July 4155-4161.

[7] Devasia, S., Chen, D., and Paden, B. (1996). "Nonlinear inversionbased output tracking", IEEE Transactions on Automatic Control, 41(7), 930-942.

[8] Boekfah, A., and Devasia, S. (2016). "Output-boundary regulation using event-based feedforward for nonminimum-phase systems", IEEE Transactions on Control Systems Technology, 24(1), 265-275.

[9] Boerlage, M. (2006). "MIMO jerk derivative feedforward for motion systems", in American Control Conference, pp. 3892-3897.

[10] Ronde, M. J. C., Schneiders, M. G. E., Kikken, E. J. G. J., Van De Molengraft, M. J. G., and Steinbuch, M. (2014). "Model-based spatial feedforward for over-actuated motion systems", Mechatronics, 24(4), 307-317.

[11] Ronde, M., van den Bulk, J., van de Molengraft, M.J.G.R., and Steinbuch, M. (2013). "Feedforward for flexible systems with timevarying performance locations", in American Control Conference, 2013, vol. 23, no. 4, , pp. 6033-6038.

[12] Kasemsinsup, Y., Heertjes, M., Butler, H., and Weiland, S. (2016) "Exact Plant Inversion of Flexible Motion Systems with a TimeVarying State-To-Output Map", in 2016 European Control Conference.

[13] Kontaras, N., Heertjes, M.F., and Zwart, H. (2016). "Continuous compliance compensation of position-dependent flexible structures", 12th IFAC International Workshop on Adaptation and Learning in Control and Signal Processing.

[14] Oomen, T., van Herpen, R., Quist, S., van de Wal, M., Bosgra, O., and Steinbuch, M. (2014). "Connecting System Identification and Robust Control for Next-Generation Motion Control of a Wafer Stage", IEEE Transactions on Control Systems Technology, Vol. 22, No. 1. 